(C) Österreichische Apotheker-Verlagsgesellschaft m. b. H, Wien, Printed in Austria

Darstellung und Atropisomerie von 1-[2-(2,2-Dimethylpiperidino)phenyl]ethanol

H. Möhrle ${ }^{\star s}$ und M. Jeandrée

Institut für Pharmazeutische Chemie der Heinrich-Heine-Universität,

Universitätsstr. 1, D-40225 Düsseldorf

Preparation and Atropisomerism of 1-[2-(2,2-Dimethylpiperidin-1-yl)phenyl]ethanol

The title compound was prepared by $\mathrm{Hg}$ (II)-edta dehydrogenation of the aminoalkohol 2 via the benzoxazine 4, which reacted with methylmagnesiumiodide to 6 . This compound shows in the NMR spectrum atropisomerism for the restricted rotation of the piperidine moiety about the aryl-C-N bond. The dehydrogenation of 6 stopped after a two electron withdrawal generating the benzoxazine 7 , because the angular hydrogen atom occupies an equatorial position, which prevents stereoelectronic conditions for a further oxidation.

(Keywords: $H g(I I)$-edta dehydrogenation, intramolecular aminoalkylation, pyrido[1,2-a][3,1]benzoxazine)

\title{
Einleitung
}

Vor kurzem berichteten wir über die Atropisomerie bei 1-(2-Aryl)piperidin-2-onen, die sich NMRspektroskopisch eindeutig nachweisen lie $\beta^{1}$. Dabei waren allerdings die Rotationsbarrieren um die Aryl-C-N-Bindung nicht hoch genug, um die Isolierung von stabilen Atropisomeren bei Raumtemperatur zu ermöglichen. Deshalb sollten 2-disubstituierte Piperidinderivate dargestellt werden, um ein "Durchdrehen" des Heterocyclus zu erschweren.

\section{Ergebnisse und Diskussion}

Die Darstellung der Verbindungen ging aus von dem Acetophenonderivat $\mathbf{1}^{2}$, das mit Lithiumaluminiumhydrid zum Aminoalkohol 2 reduziert wurde. Durch Quecksilber(II)-EDTA erfolgte unter 2-Elektronenentzug die Dehydrierung weitgehend selektiv in Richtung zum tertiären Kohlenstoff ${ }^{\beta}$ zu der thermodynamisch stabileren Carbenium-Iminiumverbindung 3, die anschließend eine intramolekulare Mannichreaktion zum BenzoxazinDerivat 4 einging. Dieses reagierte als verkappte Iminium-olat-Struktur 5 mit Methylmagnesiumiodid zum 2-geminalen Dimethylprodukt 6. Die nachfolgende Dehydrierung mit $\mathrm{Hg}(\mathrm{II})$-EDTA von 6 musste nunmehr zur sekundären $\alpha$-Methylengruppe des Stickstoffs verlaufen und ergab konsekutiv unter nucleophilem Angriff der Alkoholgruppe an die Iminiumstruktur das Benzoxazin 7. Dass die Reaktion - auch mit überschüssigem

\footnotetext{
${ }^{5}$ Herrn Univ.-Prof. Dr. W. Wendelin zum 60. Geburtstag gewidmet
} 
Oxidationsreagens - auf dieser Stufe sistierte, überraschte. Grundsätzlich könnte nämlich eine erneute Dehydrierung an der angulären Methingruppe eintreten, so dass insgesamt ein 4-Elektronenentzug unter Generierung der cyclischen Iminiumverbindung 8 resultieren sollte. Durch Umsetzung mit Wasser würde 8 schließlich unter Ringspaltung zum entsprechenden Piperidon 9 führen.<smiles>CC(=O)c1ccccc1N1CCCCC1C</smiles><smiles>CC(O)c1ccccc1N1CCCCC1C</smiles>

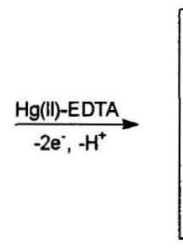<smiles>CC1=[N+](c2ccccc2C(C)O)CCCC1</smiles><smiles>[3H][IH+]</smiles><smiles>CC(O)c1ccccc1N1CCCCC1(C)C</smiles><smiles>CCCC</smiles><smiles>CC1=[N+](c2ccccc2C(C)[O-])CCCC1</smiles><smiles>CCCC</smiles><smiles>C[C@H]1O[C@]2(C)CCCCN2c2ccccc21</smiles>

$H g(I I)-E D T A \mid-2 e^{-i}-H^{+}$<smiles>C[C@H]1OC2CCCC(C)(C)N2c2ccccc21</smiles><smiles>[H][R]([H])=C1CCCCC1</smiles>

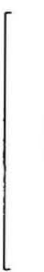<smiles>C[C@H]1OC2=NC(C)(C)CCCC2=Nc2ccccc21</smiles><smiles>[BH2-]C</smiles><smiles>CC(O)c1ccccc1N1C(=O)CCCC1(C)C</smiles>

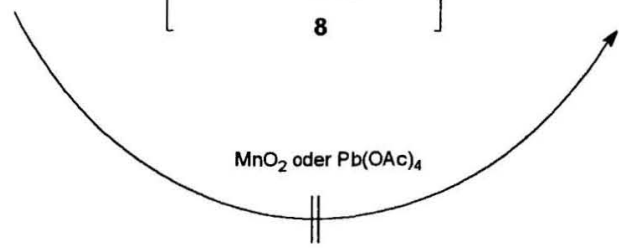

9

Diese Reaktionsfolge war tatsächlich bei der $\mathrm{Hg}(\mathrm{II})$-EDTA-Dehydrierung von dem zu 6 stellungsisomeren 4-geminalen Dimethyl-Aminoalkohol' beobachtet worden, der mit $61 \%$ Ausbeute das Lactam 10 ergab. 
Allerdings zeigte sich bereits dort, dass durch die Substitution des Piperidinrings die Ausbeute im Vergleich zum unsubstituierten Derivat deutlich abnimmt. Es ist deshalb bei 6 die Verstärkung dieses overcrowding Effekts durch die geminale Substitution in der Nähe des Stickstoffatoms zu diskutieren. Dieser schränkt nicht nur die Beweglichkeit des Heterocyclus ein, sondern kann auch durch sterische Hinderung den Angriff des Quecksilber-Komplexes am Stickstoffatom verhindern.

Eine andere Erklärung des Ausbleibens der weiteren Dehydrierung von 7 liegt in dem Mechanismus der Umsetzung, der stereoelektronische Bedingungen erfordert. Das heißt im N-mercurierten Komplex läuft die Reaktion nur dann ab, wenn das zu eliminierende Wasserstoffatom trans-diaxial zum quecksilberorganischen Rest angeordnet ist. Liegt eine cis-Verknüpfung der Ringe in $\mathbf{7}$ vor, so ist damit auch der anguläre Methinwasserstoff cis-ständig zum Quecksilbersubstituenten und damit die Umsetzung ausgeschlossen.

Es wurde deshalb versucht, mit weniger spezifischen Oxidantien, wie aktiviertem Mangan(IV)-oxid oder Bleitetraacetat, das Benzoxazin 7 zu dem Lactam 9 umzusetzen, jedoch ohne Erfolg.

\section{Spektroskopische Untersuchungen}

\section{a) Aminoalkohole}

Während der Aminoalkohol 2 mit zwei Asymmetriezentren im ${ }^{13} \mathrm{C}-\mathrm{NMR}$-Spektrum erwartungsgemäß einen doppelten Kernsatz und damit ein klassisches Diastereomerengemisch anzeigt, kann die Verdopplung der ${ }^{13} \mathrm{C}$-NMR-Signale auch bei der geminalen Dimethylverbindung 6 mit nur einem Chiralitätszentrum beobachtet werden. Daraus folgt, dass bei 6 Atropdiastereomere vorliegen, während bei 2 keine Rotationsbehinderung vorhanden ist, denn sonst hätte dort ein vierfacher Kernsatz auftreten müssen. Der in $\alpha$-Position des Piperidinrings geminal dimethylierte Aminoalkohol 6 weist somit hinsichtlich der Rotationshinderung Ähnlichkeiten mit dem 2-Piperidon $10^{1}$ auf.

Um auszuschließen, dass nur eine intramolekulare Wasserstoffbrücke für das spektroskopische Verhalten verantwortlich ist, wurden die NMR-Spektren außer in $\mathrm{CDCl}_{3}$ auch in dem stark polaren $\left[\mathrm{D}_{6}\right] \mathrm{DMSO}$ vermessen, wobei letzteres Lösungsmittel Wasserstoffbrückenbindungen brechen kann. Tatsächlich sind aber in diesem Solvens ebenso zwei Spezies im gleichen Verhältnis wie bei $\mathrm{CDCl}_{3}$ vorhanden, so dass die Atropisomerie gesichert ist.

Die Zuordnung der Signale in 6A/6B kann für die geminalen Methylgruppen allein anhand des Protonenresonanzspektrums nicht vorgenommen werden. Erst durch das ${ }^{13} \mathrm{C}-\mathrm{NMR}$-Spektrum, in dem sich die entsprechenden Signale um etwa 10 ppm unterscheiden, ist dies zu realisieren. Bei Chinolizidinderivaten mit zum Stickstoff $\alpha$-ständiger Methylgruppe ${ }^{5,6}$ zeigte sich, dass das freie Elektronenpaar des Stickstoffs zu einer Abschirmung beim axialen Methyl führt und deshalb das equatoriale Methyl bei tieferem Feld in Resonanz tritt. Dies ist auch hier zu erwarten, so dass aus der ${ }^{1} \mathrm{H}_{-}{ }^{13} \mathrm{C}-\mathrm{NMR}-$ Korrelation die zugehörigen Protonensignale hervorgehen. 
"S*, R*Atrop"
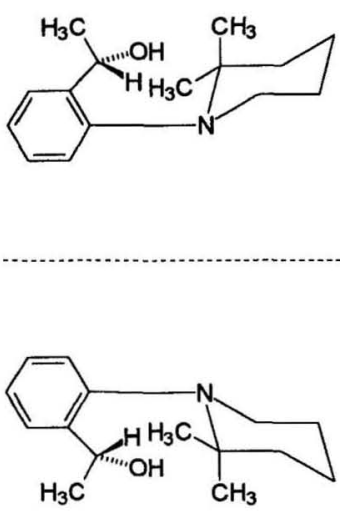

"R*, S*Atrop"
"R*, R*Atrop"

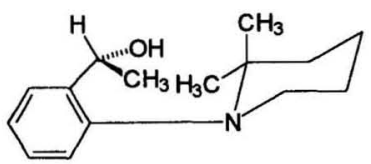

"S*, S*Atrop"

Diastereomeren-Racemate 6A/6B<smiles>CC(O)c1ccccc1N1CCC(C)(C)CC1=O</smiles>

Grundsätzlich ist bekannt, dass die Rotation bei N-Aryl-Aminen weniger gehindert ist als bei entsprechenden Amiden $^{7}$. Dies kann durch unsere Befunde auch für heterocyclische Amine und Lactame mit einer orthoständigen alkoholischen Funktion bestătigt werden. Bei solchen 2-Piperidonen' tritt bereits Rotationsbehinderung in einem solchen Ausmaß auf, dass Atropisomerie nachzuweisen ist. Dagegen ist weder bei dem C-unsubstituierten Piperidin noch beim 2-Methylpiperidinoderivat eine Rotationseinschränkung spektroskopisch sichtbar. Erst beim 2-geminalen Dimethylpiperidinoderivat kommt es zur Atropisomerie. Besonders gut ist diese nachzuweisen, wenn durch ein chirales Zentrum im Piperidonring oder in der alkoholischen Funktion Atropdiastereomere' gebildet werden. 


\section{b) Benzoxazinderivate}

Das 'H-NMR-Spektrum läßt aufgrund der Kopplungskonstanten von $4 \mathrm{a}-\mathrm{H}$ an der Verknüpfungsstelle Aussagen über die Konformation des Oxachinolizidinsystems zu. In dem Benzoxazin $\mathbf{1 2}^{3}$ als Vergleichssubstanz tritt das anguläre Proton bei $\delta_{H}=4,49$ in Resonanz, wobei das Doppeldublett mit den Kopplungskonstanten von 9,3 und $3,6 \mathrm{~Hz}$ eindeutig nachweist, dass das Methinwasserstoffatom die axiale Lage einnimmt und deshalb eine trans-Verknüpfung der Ringe vorliegt. Bei dem Hauptdiastereoisomer 7A erscheint das Signal bei $\delta_{\mathrm{H}}=4,57$, dagegen fehlt aber eine große axial/axial-Kopplung, und die beiden kleinen Kopplungen von jeweils $2,7 \mathrm{~Hz}$ zeigen ein equatoriales Wasserstoffatom am Brückenkopf und damit die Konformation cis/eq an. Dabei ist davon auszugehen, daß aufgrund von 1,3-diaxialen Wechselwirkungen in 7A der Piperidinring nicht in der idealen Sesselform vorliegt. Eine Aussage über die Konformation des Nebendiastereomers 7B (27\%) ist anhand der Signalform und der Kopplungen nicht möglich wegen der Überlagerung des Signals des angulären Protons durch das des Hauptdiastereomers 7A. Indessen indiziert die Gesamtsignalbreite beider Diastereomere von etwa $10 \mathrm{~Hz}$ ebenfalls eine cis/eq Konformation für 7B, so daß der Unterschied der Diastereomere nur aus der Stellung der 6-Methylgruppe resultiert. Das erklärt auch die Resistenz von 7A und 7B gegen eine weitere $\mathrm{Hg}$ (II)-EDTA-Dehydrierung, weil die stereoelektronischen Voraussetzungen fehlen.

Durch einen ${ }^{13} \mathrm{C}$-NMR-Datenvergleich (Tab.1) sollten die Konformationen bestätigt oder nachgewiesen werden. Die Zuordnung der Signallagen von 4 A und 7A/7B erfolgte durch Interpretation von ${ }^{1} \mathrm{H}-{ }^{13} \mathrm{C}$ korrelierten NMR-Spektren zusammen mit den für Methylgruppen bekannten $\beta$ - und $\gamma$-Effekten. Wenn eine axialständige Methylgruppe am angulären C-4a und damit eine trans-Verknüpfung der Ringe in $4 \mathrm{~A}$ vorläge, soltte diese aufgrund des $\gamma$-Effekts einen deutlichen Hochfeldshift an C-1 hervorrufen. Dieser ist jedoch im Vergleich zu der nicht methylierten trans-verknüpften Verbindung 12 nicht signikant. Deshalb folgt hieraus die equatoriale Position der Methylgruppe am C-4a verbunden mit der cis/eq Konformation von 4A. Bereits früher wurde beobachtet ${ }^{8}$, daß ein axialständiges Sauerstoffatom einen - wenn auch vergleichsweise zur Methylgruppe - geringeren $\gamma$-Effekt ausübt. Allein auf diesen ist der geringe Hochfeldshift von 2,3 ppm für C-1 in 11 gegenüber dem transverknüpften 12 (mit equatoriaiem Sauerstoffatom) zurückzuführen.

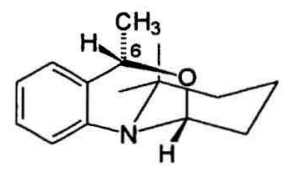

7A

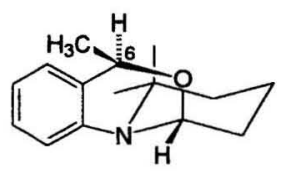

78

Beim Vergleich der Diastereomerenpaare 7A und 7B fält ein $\gamma$-Effekt von 4,86 ppm auf C-4a auf, der nur von der C-6-Methylgruppe herrühren kann. Damit sollte die Methylgruppe an C-6 in 7A equatorial und in 7B axial stehen. 
Tab. 1: Ausgewählte ${ }^{13} \mathrm{C}$-NMR-Daten $\left(\mathrm{CDCl}_{3} ; \delta[\mathrm{ppm}]\right)$ von $4 \mathrm{~A}, 11,12$ und 7

\begin{tabular}{|c|c|c|c|c|c|}
\hline C-Atom & $\begin{array}{l}\mathrm{CH}_{3} \\
4 \mathrm{~A}^{*}\end{array}$ & $11^{3}$ & $12^{3}$ & \multicolumn{2}{|c|}{$\begin{array}{l}\mathrm{CH}_{3} \\
\quad 7 \mathrm{~B}\end{array}$} \\
\hline$C-1$ & 44.33 & 42.9 & 45.2 & 55.17 & 55.96 \\
\hline $1-\mathrm{CH}_{3}$ eq & - & - & - & 34.26 & 33.99 \\
\hline $1-\mathrm{CH}_{3}$ ax & - & - & - & 24.36 & 23.26 \\
\hline$C-4$ & 37.99 & 37.4 & 31.5 & 30.85 & 30.69 \\
\hline$C-4 a$ & 86.72 & 85.2 & 85.9 & 84.90 & 80.04 \\
\hline $4 \mathrm{a}-\mathrm{CH}_{3}$ & 17.30 & 18.2 & - & - & - \\
\hline$C-6$ & 66.91 & 61.4 & 66.7 & 73.21 & 70.22 \\
\hline $6-\mathrm{CH}_{3}$ & 22.14 & - & - & 22.00 & 23.07 \\
\hline
\end{tabular}


Dem Fonds der Chemischen Industrie danken wir für die Unterstützung dieser Arbeit.

\section{Experimenteller Teil}

Schmelzpunkte (unkorrigiert): Linström-Block. Siedepunkte: Kugelrohr Büchi GKR-50, Luftbadtemperatur. IR: Perkin-Elmer 177 und FT 1600. MS: Finigan 3500, lonisierungsenergie $70 \mathrm{eV}$. ${ }^{1} \mathrm{H}$ - und ${ }^{13} \mathrm{C}-\mathrm{NMR}$ : Varian FT-80A und Bruker AC 200F, Tetramethyisilan als innerer Standard, $\delta$ in ppm. Präparative Schichtchromatographie: PSC-Fertigplatten Kieselgel $60 \mathrm{~F}_{254 \mathrm{~s}}$ mit Konzentrierungszone, $1 \mathrm{~mm}$ oder $2 \mathrm{~mm}$ (Merck) Weitere exp. Details, insbesondere spektroskopische Daten vgl. Lit.?

\section{1-[2-(2-Methylpiperidino)phenyl]ethanol (2)}

$5.05 \mathrm{~g}(23.25 \mathrm{mmol}) 2^{\prime}$-(2-Methylpiperidino)acetophenon $(1)^{2}$ werden in absolutem Ether mit $1.76 \mathrm{~g}$ (46.50 mmol) Lithiumaluminiumhydrid $1 \mathrm{~h}$ unter Rückfluss erhitzt. Nach Hydrolyse, üblicher Aufarbeitung und Destillation resultiert ein Öl vom Sdp. $160^{\circ} \mathrm{C} / 0.014$ Torr. Ausbeute: $4.9 \mathrm{~g}(96 \%)$. - IR (Film, cm $\left.\mathrm{cm}^{-1}\right): 3402$ br, $3062,3028,2969,2934,2855,1598,1578,1486$. Eine Verdünnungsreihe in Tetrachlorkohlenstoff weist eine intramolekulare Wasserstoffbrücke nach. - $M S\left(35^{\circ} \mathrm{C}\right): \mathrm{m} / \mathrm{z}(\%)=219\left(28 ; \mathrm{M}^{+}\right), 204(52), 186(20), 148(100)$, $130(56), 77(80)$. - 'H-NMR ( $\left.\mathrm{CDCl}_{3}\right): \delta(\mathrm{ppm}) ; \mathrm{J}(\mathrm{Hz})=8.16(\mathrm{~s} \mathrm{br}, 0.6 \mathrm{H}, \mathrm{OH}[2 \mathrm{~A}]$, aust $), 7.83(\mathrm{~s} \mathrm{br}, 0.4 \mathrm{H}, \mathrm{OH}$ [2B], aust.), 7.30-7.08 (m, 4H, aromat. $\mathrm{H}), 5.14\left(\mathrm{q}, 0.4 \mathrm{H}, 1-\mathrm{H}[2 \mathrm{~B}],{ }^{3} \mathrm{~J}=6.5\right), 5.02\left(\mathrm{q}, 0.6 \mathrm{H}, 1-\mathrm{H}[2 \mathrm{~A}],{ }^{3} \mathrm{~J}=\right.$ 6.6), 3.10-2.91 (m, 2H, 6"-- $\left.\mathrm{H}_{\text {eq }}, 2^{\prime \prime}-\mathrm{H}\right), 2.78-2.59\left(\mathrm{~m}, 1 \mathrm{H}, 6 "-\mathrm{H}_{\mathrm{ax}}\right), 1.86-1.39\left(\mathrm{~m}, 6 \mathrm{H}, 3 "-\mathrm{H}_{2}, 4^{\prime \prime}-\mathrm{H}_{2}, 5^{\prime \prime}-\mathrm{H}_{2}\right), 1.55$ (d, $\left.1.2 \mathrm{H}, 1-\mathrm{CH}_{3}[2 \mathrm{~B}],{ }^{3} \mathrm{~J}=6.5\right), 1.51\left(\mathrm{~d}, 1.8 \mathrm{H}, 1-\mathrm{CH}_{3}[2 \mathrm{~A}],{ }^{3} \mathrm{~J}=6.6\right), 0.93\left(\mathrm{~d}, 1.2 \mathrm{H}, 2^{\prime \prime}-\mathrm{CH}_{3}[2 \mathrm{~B}],{ }^{3} \mathrm{~J}=6.4\right), 0.88$ (d, $1.8 \mathrm{H}, 2 "-\mathrm{CH}_{3}[2 \mathrm{~A}],{ }^{3} \mathrm{~J}=6.2$ ). Aus der Integration über $1-\mathrm{H}, \mathrm{OH}$ und $2 "-\mathrm{CH}_{3}$ in $\mathrm{CDCl}_{3}$ errechnet sich ein Diastereomerenverhältnis 2A:2B von 60\%:40\%. - 'H-NMR (DMSO- $\left.\mathrm{d}_{6}\right): \delta(\mathrm{ppm}) ; \mathrm{J}(\mathrm{Hz})=7.47-7.41(\mathrm{~m}, 1 \mathrm{H}$,

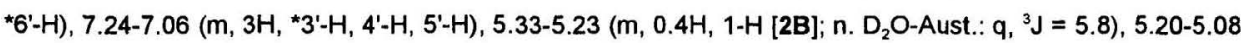
$\left(\mathrm{m}, 1.6 \mathrm{H}, 1-\mathrm{H}[2 \mathrm{~A}] ; \mathrm{OH}\right.$, aust.; $n$. $\mathrm{D}_{2} \mathrm{O}-A$ ust.: $\left.\mathrm{q},{ }^{3} \mathrm{~J}=5.8\right), 3.03-2.37\left(\mathrm{~m}, 3 \mathrm{H}, 66^{\prime \prime}-\mathrm{H}_{2}, 2^{\prime \prime}-\mathrm{H}\right), 1.77-1.15(\mathrm{~m}, 6 \mathrm{H}$, 3"- $\mathrm{H}_{2}, 4^{\prime \prime}-\mathrm{H}_{2}, 5$ "- $\left.\mathrm{H}_{2}\right), 1.27$ ('t', 3H, 1-CH $\mathrm{CH}_{3}$ 'J' = 5.8), $0.75\left(\mathrm{~d}, 1.8 \mathrm{H}, 2 "-\mathrm{CH}_{3}[2 \mathrm{~A}],{ }^{3} \mathrm{~J}=4.8\right), 0.72\left(\mathrm{~d}, 1.2 \mathrm{H}, 2 "-\mathrm{CH}_{3}\right.$ $[2 \mathrm{~B}],{ }^{3} \mathrm{~J}=4.6$ ). Aus der Integration über 1-H in DMSO- $\mathrm{d}_{6}$ errechnet sich ein Diastereomerenverhältnis 2A:2B von 60\%:40\%. - ${ }^{13} \mathrm{C}-\mathrm{NMR}\left(\mathrm{CDCl}_{3}\right)$ : $\delta$ (ppm) 2A/2B = 149.46/149.57 (C-2'); $141.63 / 141.17$ (C-1'); $127.52 / 127.52\left({ }^{*} \mathrm{C}-4^{\prime}\right) ; 127.40 / 126.67\left({ }^{*} \mathrm{C}-6^{\prime}\right) ; 125.83 / 125.54\left({ }^{*} \mathrm{C}-5^{\prime}\right), 123.91 / 123.32\left({ }^{*} \mathrm{C}-3^{\prime}\right) ; 71.03 / 68.68$ (C-1), 57.80/57.80 (C-2"); 57.27/56.80 (C-6"); 30.06/30.06 (C-3"); 26.77/26.77 (“C-5"); 26.45/23.75 (1-CH ( $_{3}$; 24.71/24.71 ("C-4"); 20.30/20.30 (2"- $\left.\mathrm{CH}_{3}\right)$. Die Zuordnung erfolgt anhand von ' $\mathrm{H}-{ }^{13} \mathrm{C}-$ korrelierten Spektren. $\mathrm{C}_{14} \mathrm{H}_{21} \mathrm{NO}$ (219.3): Ber. C 76.67 H 9.65 N 6.39; Gef. C 76.75 H 9.77 N 6.09 .

\section{4a,6-Dimethyl-2,3,4,4a-tetrahydro-1H,6H-pyrido[1,2-a][3, 1]benzoxazin (4)}

8 Oxidations-Äquivalente gelbes Quecksilber(II)-oxid und die äquimolare Menge Dinatriumethylendiamintetraacetat-Dihydrat werden in $40 \mathrm{ml}$ Ethanol/Wasser (1+1) bis zur Lösung gerührt. Dann wird $1.00 \mathrm{~g}$ (4.56 mmol) 2 zu der im kochenden Wasserbad erwärmten und mit Stickstoff begasten Hg(II)-EDTA-Lösung gegeben und unter Rückfluss $1 \mathrm{~h}$ weiter erhitzt. Nach Abkühlung wird abfiltriert, der Rückstand mit Ethanol 
und Methylenchlorid gewaschen und das verbleibende Quecksilber gravimetrisch bestimmt $[0.91 \mathrm{~g} \mathrm{(100 \%}$ bez. auf 2 Oxid.-Äquiv.)]. Die organischen Lösungsmittel werden i. Vak. entfernt, die wässrige Lösung mit konz. Ammoniak alkalisiert und erschöpfend mit Methylenchlorid extrahiert. Die organischen Phasen werden über Natriumsulfat getrocknet und i. Vak. eingeengt. Die leicht gelbliche Rohbase wird durch präparative Schichtchromatographie mit Methylenchlorid/Isopropanol $(100+2.5)$ [Rf-Wert: 0.48] gereinigt: Amorpher, weißer, wachsartiger Feststoff (Aufbewahrung im Kühlschrank) zersetzt sich bei Raumtemperatur und Tageslicht unter Rotfärbung. Schmp. 33-34 ${ }^{\circ} \mathrm{C}$. Ausbeute: $760 \mathrm{mg}$ (77\%). - IR (Film, cm${ }^{-1}$ ): 3071, 3032, 2979, 2939, 2867, 1604, 1578, 1492. - MS $\left(30^{\circ} \mathrm{C}\right): \mathrm{m} / \mathrm{z}(\%)=217\left(13 ; \mathrm{M}^{+}\right), 202(100), 146(22), 132(26), 118(13)$, 91 (18), 77 (44). - 'H-NMR (CDCl $)$ ): $\delta$ (ppm); J (Hz) = 7.20-7.06 ('ddd', $1 \mathrm{H}, 9-\mathrm{H},{ }^{3} \mathrm{~J}=7.7,{ }^{3} \mathrm{~J}=7,{ }^{4} \mathrm{~J}=1.4$ ), $6.98\left(' d ', 1 \mathrm{H}, 7-\mathrm{H}, \mathrm{J}^{\mathrm{J}}=7\right), 6.87-6.71(\mathrm{~m}, 2 \mathrm{H}, 8-\mathrm{H}, 10-\mathrm{H}), 5.06\left(\mathrm{q}, 0.87 \mathrm{H}, 6-\mathrm{H}[4 \mathrm{~A}],{ }^{3} \mathrm{~J}=6.4\right), 4.72-4.62(\mathrm{~m}$, $0.13 \mathrm{H}, 6-\mathrm{H}$ [4B, 4C, 4D], 4.16-4.03 (m, 0.065H, 1- $\mathrm{H}_{\mathrm{eq}}$ [4C?+4D?]), 3.56 ('dt', 0.935H, 1- $\mathrm{H}_{\mathrm{eq}}[4 \mathrm{~A}+4 \mathrm{~B}$ ?], ' $\mathrm{J}$ ' = 12.2, 'J' = 3, 'J' = 3), 3.02-2.84 (m, 0.065H, 1- $\mathrm{H}_{\mathrm{ax}}\left[4 \mathrm{C} ?+4 \mathrm{D}\right.$ ?]), 2.65 ('ddd', 0.935H, 1- $\mathrm{H}_{\mathrm{ax}}[4 \mathrm{~A}+4 \mathrm{~B}$ ?], 'J' = 12.2, ' $\mathrm{J}$ ' = 3.3, ' $\mathrm{J}$ ' = 3), $1.97-1.40\left(\mathrm{~m}, 6 \mathrm{H}, 2-\mathrm{H}_{2}, 3-\mathrm{H}_{2}, 4-\mathrm{H}_{2}\right), 1.55\left(\mathrm{~d}, 3 \mathrm{H}, 6-\mathrm{CH}_{3},{ }^{3} \mathrm{~J}=6.4\right), 1.30\left(\mathrm{~s}, 2.61 \mathrm{H}, 4 \mathrm{a}-\mathrm{CH}_{3}\right.$ [4A]), $1.15\left(\mathrm{~s}, 0.195 \mathrm{H}, 4 \mathrm{a}-\mathrm{CH}_{3}[4 \mathrm{~B}]\right), 1.08\left(\mathrm{~s}, 0.0975 \mathrm{H}, 4 \mathrm{a}-\mathrm{CH}_{3}[4 \mathrm{C}]\right), 1.05\left(\mathrm{~s}, 0.0975 \mathrm{H}, 4 \mathrm{a}-\mathrm{CH}_{3}\right.$ [4D]). Aus der Integration über die 4a- $\mathrm{CH}_{3}$-Gruppe in $\mathrm{CDCl}_{3}$ errechnet sich für die Diastereomere $4 \mathrm{~A}, \mathbf{4 B}, \mathbf{4 C}$ und $4 \mathrm{D}$ ein Verhăltnis von ungefähr $87 \%: 6.5 \%: 3.25 \%: 3.25 \% .{ }^{13} \mathrm{C}-\mathrm{NMR}\left(\mathrm{CDCl}_{3}\right): \delta(\mathrm{ppm}) \mathbf{4 A}(87 \%)=143.73(\mathrm{C}-10 \mathrm{a})$; 127.71 (C-6a); 127.39 (C-9); 124.54 (C-7); 118.47 (C-8), 113.75 (C-10); 86.72 (C-4a), 66.91 (C-6); 44.33 (C-1); 37.99 (C-4); $25.28\left({ }^{\star} \mathrm{C}-2\right) ; 22.14\left(6-\mathrm{CH}_{3}\right) ; 21.04\left({ }^{\star} \mathrm{C}-3\right) ; 17.30\left(4 \mathrm{a}-\mathrm{CH}_{3}\right)$. Die Zuordnung erfolgt anhand von ${ }^{1} \mathrm{H}-{ }^{13} \mathrm{C}$-korrelierten Spektren. Nicht zuordnungsfähige Signale für 4B, 4C, 4D: 128.50; 127.77; 124.71; 122.36; 118.56; 114.97; 112.95; 86.72; 82.41; 72.54; 65.53; 48.40; 44.21; 37.90; 36.94; 32.13; 31.02; 25.44; $22.31 ; 21.44 ; 20.66 ; 18.55 ; 17.52 ; 13.90 . \mathrm{C}_{14} \mathrm{H}_{19} \mathrm{NO}(217.3)$ : Ber. C 77.38 H 8.81 N $6.45 ;$ Gef. C $77.36 \mathrm{H}$ 8.84 N 6.62 .

\section{1-[2-(2,2-Dimethylpiperidino)phenyl]ethanol (6)}

Zu dem Grignard-Reagens aus $0.36 \mathrm{~g}$ (15 mmol) Magnesiumspänen und $2.13 \mathrm{~g}$ (15 mmol) Methyliodid in siedendem absolutem Ether wird innerhalb $15 \mathrm{~min}$ eine Lösung von $1.30 \mathrm{~g}(6 \mathrm{mmol}) 4$ in Ether zugetropft. Nach weiteren 135 min Reflux erfolgt Hydrolyse mit Ammoniumchlorid-Lösung. Die erhaltene Base wird im Kugelrohr destilliert, durch präparative Schichtchromatographie $(2 \mathrm{~mm})$ mit Ether (Rf-Wert: 0.53$)$ gereinigt und redestilliert: Farbloses Öl. Sdp. $200^{\circ} \mathrm{C}$ (Luftbad)/0.016 Torr. Ausbeute: $750 \mathrm{mg}(54 \%)$. IR (Film, $\left.\mathrm{cm}^{-1}\right)$ : $3401,3063,3027,2973,2932,2865,1596,1577,1484$. $-M S\left(50^{\circ} \mathrm{C}\right): \mathrm{m} / \mathrm{z}(\%)=233\left(17 ; \mathrm{M}^{+}\right), 218(44), 200$ (100), 148 (78), 144 (49), 132 (53), 130 (48), 91 (23), 77 (67). - 'H-NMR ( $\left.\mathrm{CDCl}_{3}\right): \delta(\mathrm{ppm}) ; \mathrm{J}(\mathrm{Hz})=8.97(\mathrm{~s}$ $\mathrm{br}, 0.7 \mathrm{H}, 1-\mathrm{OH}$ [6A], aust.), 8.06 (s br, 0.3H, 1-OH [6B], aust.), 7.27-7.13 (m, 4H, 3'-H, 4'-H, 5'-H, 6'-H), 5.25 $\left(q, 0.3 \mathrm{H}, 1-\mathrm{H}[6 \mathrm{~B}],{ }^{3} \mathrm{~J}=6.4\right), 5.22\left(\mathrm{q}, 0.7 \mathrm{H}, 1-\mathrm{H}[6 \mathrm{~A}],{ }^{3} \mathrm{~J}=6.4\right), 3.28-3.15\left(\mathrm{~m}, 1 \mathrm{H},{ }^{*} 6 "-\mathrm{H}_{\mathrm{ax}}\right), 2.83-2.76(\mathrm{~m}, 1 \mathrm{H}$, "6"- $\left.\mathrm{H}_{\text {eq }}\right), 1.76-1.51\left(\mathrm{~m}, 6 \mathrm{H}, 3 "-\mathrm{H}_{2}, 4 "-\mathrm{H}_{2}, 5 "-\mathrm{H}_{2}\right), 1.54\left(\mathrm{~d}, 2.1 \mathrm{H}, 1-\mathrm{CH}_{3}[6 \mathrm{~A}],{ }^{3} \mathrm{~J}=6.4\right), 1.53\left(\mathrm{~d}, 0.9 \mathrm{H}, 1-\mathrm{CH}_{3}\right.$ $\left.[6 \mathrm{~B}],{ }^{3} \mathrm{~J}=6.4\right), 1.28\left(\mathrm{~s}, 0.9 \mathrm{H}, 2^{2}-\mathrm{CH}_{3} \mathrm{ax}[6 \mathrm{~B}]\right), 1.22\left(\mathrm{~s}, 2.1 \mathrm{H}, 2^{2}-\mathrm{CH}_{3} \mathrm{ax}[6 \mathrm{~A}]\right), 1.04\left(\mathrm{~s}, 0.9 \mathrm{H}, 2^{\prime \prime}-\mathrm{CH}_{3} \mathrm{eq}[6 \mathrm{~B}]\right)$, $1.00\left(\mathrm{~s}, 2.1 \mathrm{H}, 2 "-\mathrm{CH}_{3}\right.$ eq [6A]). Aus der Integration über 2"- $\mathrm{CH}_{3} \mathrm{eq}$ in $\mathrm{CDCl}_{3}$ errechnet sich ein Verhältnis 6A:6B von 70\%:30\%. - 'H-NMR (DMSO- $\left.\mathrm{d}_{6}\right): \delta(\mathrm{ppm}) ; \mathrm{J}(\mathrm{Hz})=7.54-7.45\left(\mathrm{~m}, 0.7 \mathrm{H},{ }^{*}{ }^{\prime}{ }^{\prime}-\mathrm{H}[6 \mathrm{~A}]\right), 7.42-7.36(\mathrm{~m}$, 
$\left.0.3 \mathrm{H},{ }^{\prime \prime} 6^{\prime}-\mathrm{H}[6 \mathrm{~B}]\right), 7.26-7.10\left(\mathrm{~m}, 3 \mathrm{H}, 3^{\prime}-\mathrm{H}, 4^{\prime}-\mathrm{H}, 5^{\prime}-\mathrm{H}\right), 5.49-5.41\left(\mathrm{~m}, 0.7 \mathrm{H}, 1-\mathrm{H}[6 \mathrm{~A}], \mathrm{n} . \mathrm{D}_{2} \mathrm{O}-A u s t .: q^{3}{ }^{3} \mathrm{~J}=6\right)$, 5.38-5.27 (m, 0.3H, 1-H [6B], n. $D_{2} \mathrm{O}-A$ ust.: $q,{ }^{3} \mathrm{~J}=6$ ), 4.96 (d. $0.7 \mathrm{H}, \mathrm{OH}\left[6 \mathrm{~A}\right.$ ), aust., ${ }^{3} \mathrm{~J}=4.6$ ), 3.34 ('s',

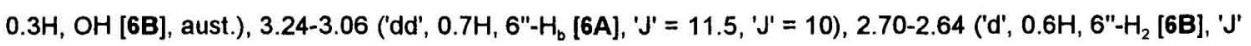
$=11.9), 2.44-2.38\left(' d ', 0.7 \mathrm{H}, 6 "-\mathrm{H}_{2}[6 \mathrm{~A}]\right.$, 'J' = 11.5), 1.74-1.56 (m, 4H, 4"- $\left.\mathrm{H}_{2}, 5 "-\mathrm{H}_{2}\right), 1.56-1.39\left(\mathrm{~m}, 2 \mathrm{H}, 3^{\prime \prime}-\mathrm{H}_{2}\right)$, $1.33\left(\mathrm{~d}, 0.9 \mathrm{H}, 1-\mathrm{CH}_{3}[6 \mathrm{~B}],{ }^{3} \mathrm{~J}=6.4\right), 1.20\left(\mathrm{~s}, 0.9 \mathrm{H}, 2 "-\mathrm{CH}_{3} \mathrm{ax}[6 \mathrm{~B}]\right), 1.18\left(\mathrm{~d}, 2.1 \mathrm{H}, 1-\mathrm{CH}_{3}[6 \mathrm{~A}],{ }^{3} \mathrm{~J}=6.4\right), 1.14$ (s, 2.1H, 2"- $\left.\mathrm{CH}_{3} \mathrm{ax}[6 \mathrm{~A}]\right), 0.83\left(\mathrm{~s}, 2.1 \mathrm{H}, 2 "-\mathrm{CH}_{3} \mathrm{eq}[6 \mathrm{~A}]\right), 0.81$ (s, 0.9H, 2"- $\mathrm{CH}_{3}$ eq [6B]). Aus der Integration über $1-\mathrm{H}$ sowie über $1-\mathrm{CH}_{3}[6 \mathrm{~B}]$ in DMSO- $\mathrm{d}_{6}$ errechnet sich ein Verhältnis $6 \mathrm{~A}: 6 \mathrm{~B}$ von $70 \%: 30 \%$. $-{ }^{13} \mathrm{C}-\mathrm{NMR}$ $\left(\mathrm{CDCl}_{3}\right): \delta(\mathrm{ppm})$ 6A/6B = 147.91/147.19 (C-2'); 142.75/142.50 (C-1'); 127.48/127.30 (C-4'); 126.74/126.50 ( $\left.{ }^{*} \mathrm{C}-6^{\prime}\right) ; 126.57 / 126.46$ ( $\left.{ }^{*} \mathrm{C}-5^{\prime}\right) ; 126.14 / 125.98\left({ }^{*} \mathrm{C}-3^{\prime}\right) ; 69.34 / 70.02$ (C-1); 56.29/55.05 (C-2"); 49.60/49.90 (C-6"); 41.21/41.46 (C-3"); 29.21/30.15 (2"- $\left.\mathrm{CH}_{3} \mathrm{eq}\right) ; 26.54 / 26.59$ (C-5"); 23.74/25.93 (1-CH $\left.{ }_{3}\right) ; 20.86 / 20.91$ (C-4"); 18.25/18.85 (2"-CH $\left.\mathrm{CH}_{3} \mathrm{ax}\right) .{ }^{13} \mathrm{C}-\mathrm{NMR}$ (DMSO-d ${ }_{6}$ ): $\delta$ (ppm) 6A/6B = 147.15/147.57 (C-2'); 146.74/142.26 (C-1'); 126.74-125.39/126.53-125.19 (C-3' bis C-6'); 64.22/63.44 (C-1); 53.86/53.80 (C-2"); 48.78/49.26 (C-6"); 41.04/41.18 (C-3"); 29.39/29.85 (2"-CH $\left.\mathrm{CH}_{3} \mathrm{eq}\right) ; 26.51 / 26.51$ (C-5"); 26.05/24.24 (1-CH $\left.\mathrm{CH}_{3}\right) ; 20.83 / 20.71$ (C-4"); 17.71/18.57 (2"-CH $\mathrm{CH}_{3}$ ax). Die Signalzuordnung erfolgt anhand ${ }^{1} \mathrm{H}-{ }^{13} \mathrm{C}-$ korrel. NMR-Spektren. $\mathrm{C}_{15} \mathrm{H}_{23} \mathrm{NO}$ (233.4): Ber. C 77.21 H 9.93 N 6.00; Gef. C 76.92 H 9.97 N 5.74.

\section{1,1,6-Trimethyl-2,3,4,4a-tetrahydro-1H,6 $\mathrm{H}$-pyrido[1,2-a][3, 1]benzoxazin (7)}

Darstellung durch $\mathrm{Hg}(\mathrm{II})$-EDTA-Dehydrierung von 6 analog zu der von 2. Ansatz: $750 \mathrm{mg}$ (3.21 mmol) 6; 8 Oxid.-Äquiv. Hg(II)-EDTA; $40 \mathrm{ml}$ Wasser; Reaktionszeit $1 \mathrm{~h}$. $\mathrm{Hg}$-Abscheidung: $1.16 \mathrm{~g}(90 \%$ bez. auf 2 Oxid.-Äquiv.). Die rotbraune Reaktionslösung wird mit Pottasche ausgesalzt, anschließend mit Methylenchlorid erschöpfend extrahiert und der Rückstand aus den vereinigten organischen Phasen wiederholt säulenchromatographisch aufgetrennt (Kieselgel, Länge $4 \mathrm{~cm}$, Durchmesser $1.5 \mathrm{~cm}$; Eluens: 1) Ether. 2) Methylenchlorid). Das gelbliche Eluat der zweiten Säule wird durch präparative Schichtchromatographie $(2 \mathrm{~mm})$ mit Methylenchlorid/Isopropanol $(100+2,5)$ gereinigt: Leicht grünliches Öl, das im Kühlschrank kristallin ist und sich bei Raumtemperatur verflüssigt. Ausbeute: $140 \mathrm{mg}(19 \%)$ - IR (Film, $\left.\mathrm{cm}^{-1}\right): 3070,3023,2949,2876,2849,1602,1574,1482 .-M S\left(30^{\circ} \mathrm{C}\right): \mathrm{m} / \mathrm{z}(\%)=231\left(21 ; \mathrm{M}^{+}\right), 216$ (100), 202 (13), 160 (37), 148 (36), 132 (19), 130 (30), 118 (35), 105 (13), 91 (28), 77 (57). - 'H-NMR $\left(\mathrm{CDCl}_{3}\right): \delta(\mathrm{ppm}) ; \mathrm{J}(\mathrm{Hz})=7.11-7.02(\mathrm{~m}, 4 \mathrm{H}, 7-\mathrm{H}, 8-\mathrm{H}, 9-\mathrm{H}, 10-\mathrm{H}), 5.11(\mathrm{q}, 0.73 \mathrm{H}, 6-\mathrm{H}[7 \mathrm{~A}], 3 \mathrm{~J}=6.5), 5.01$ $\left(q, 0.27 \mathrm{H}, 6-\mathrm{H}[7 \mathrm{~B}],{ }^{3} \mathrm{~J}=6.6\right), 4.58\left(?, 0.27 \mathrm{H}, 4 \mathrm{a}-\mathrm{H}_{\text {eq }}[7 \mathrm{~B}]\right)$, überlagert von $4.57\left(' \mathrm{t}, 0.73 \mathrm{H}, 4 \mathrm{a}-\mathrm{H}_{\text {eq }}[7 \mathrm{~A}],{ }^{3} \mathrm{~J}=\right.$ $\left.2.7,{ }^{3} \mathrm{~J}=2.7\right), 2.04-1.46\left(\mathrm{~m}, 6 \mathrm{H}, 2-\mathrm{H}_{2}, 3-\mathrm{H}_{2}, 4-\mathrm{H}_{2}\right), 1.56\left(\mathrm{~d}, 2.19 \mathrm{H}, 6-\mathrm{CH}_{3}[7 \mathrm{~A}],{ }^{3} \mathrm{~J}=6.5\right), 1.48(\mathrm{~d}, 0.81 \mathrm{H}$, $\left.6-\mathrm{CH}_{3}[7 \mathrm{~B}],{ }^{3} \mathrm{~J}=6.6\right), 1,35\left(\mathrm{~s}, 2.19 \mathrm{H}, 1-\mathrm{CH}_{3} \mathrm{ax}[7 \mathrm{~A}]\right), 1.31\left(\mathrm{~s}, 0.81 \mathrm{H}, 1-\mathrm{CH}_{3} \mathrm{ax}[7 \mathrm{~B}]\right), 1.08\left(\mathrm{~s}, 2.19 \mathrm{H}, 1-\mathrm{CH}_{3} \mathrm{eq}\right.$ [7A]), $1.00\left(\mathrm{~s}, 0.81 \mathrm{H}, 1-\mathrm{CH}_{3} \mathrm{eq}[7 \mathrm{~B}]\right)$. Aus der Integration über 1- $\mathrm{CH}_{3}$ ax und 1- $\mathrm{CH}_{3}$ eq errechnet sich ein Diastereomerenverhältnis 7A:7B von $73 \%: 27 \%$. ${ }^{13} \mathrm{C}-\mathrm{NMR}\left(\mathrm{CDCl}_{3}\right): \delta(\mathrm{ppm}) 7 \mathrm{~A} / 7 \mathrm{~B}=144.55 / 144.74(\mathrm{C}-10 \mathrm{a})$; 136.00/135.89 (C-6a); 127.77/127.69 ("C-9); 125.31/125.89 ( $\left.{ }^{*} \mathrm{C}-7\right) ; 125.19 / 125.38$ ( $\left.{ }^{*} \mathrm{C}-8\right) ; 123.81 / 125.14$ ( $\left.{ }^{*} \mathrm{C}-10\right) ; 84.90 / 80.04$ (C-4a); 73.21/70.22 (C-6); 55.17/55.96 (C-1); 39.85/39.75 (C-2); 34.26/33.99 (1-CH $\left.{ }_{3} \mathrm{eq}\right)$; $30.85 / 30.69$ (C-4); 24.36/23.26 (1- $\left.\mathrm{CH}_{3} \mathrm{ax}\right) ; 22.00 / 23.07\left(6-\mathrm{CH}_{3}\right) ; 15.80 / 15.86$ (C-3). Die Signalzuordnung 
erfolgt anhand eines ${ }^{1} \mathrm{H}-{ }^{13} \mathrm{C}$-korrel. NMR-Spektrums. $\mathrm{C}_{15} \mathrm{H}_{21} \mathrm{NO}$ (231.3): Ber. C $77.88 \mathrm{H} 9.15 \mathrm{~N} 6.05$; Gef. C 77.77 H 9.16 N 6.00 .

\section{Literatur}

1 Möhrle H., Jeandrée M., Breitmaier E., Rohonczy J. (2000), J. Prakt. Chem. 342: 473

2 Möhrle H., Mehrens J. (1998), Z. Naturforsch. 53b: 1369

3 Möhrle H., Mehrens J. (1998), Z. Naturforsch. 53b: 37

4 Möhrle H., Gehlen M. (1992), Arch. Pharm. 325: 335

5 LaLonde R. T., Donvito T. N. (1974), Can. J. Chem. 52: 3778

6 Tourwé D., Van Binst G. (1978), Heterocycles 9: 507

7 Kessler H. (1970), Angew. Chem. 82: 237

8 Möhrle H., Ottersbach D., Steigel A. (1992), Arch. Pharm. 325: 157

9 Jandrée M. (1992), Diss. Universität Düsseldorf 